\title{
Piles of Thumbnails - Visualizing Document Management
}

\author{
Matjaž Kljun \\ Faculty of Education Koper \\ University of Primorska \\ Cankarjeva 5, 6000 Koper, Slovenia \\ mkljun@pef.upr.si
}

\begin{abstract}
Digital information is present in everyone's life. Most of the time we spend in front of a computer is in managing (processing, creating, editing, searching, and organizing), digital documents. Current tools for managing digital information are based on concepts developed over 40 years ago. Even with the desktop metaphor and improved visualization, these methods do not support management of large amounts of information. This paper provides an overview of the current state of personal information management and suggests a design based on a "piles of documents" metaphor that more closely supports the working habits of many users.
\end{abstract}

Keywords. personal information management, document piles, thumbnails, visual context

\section{Introduction}

The term, "Personal Information Management" (PIM) describes the acquisition, storage, organization, and retrieval of digital information collections by an individual in their digital environment [11]. This includes files, electronic mail, bookmarks of web pages, instant messages, address book entries, and other information an individual or the computer might collect, create, get, edit, store, or otherwise manipulate.

Barreau [3] divided PIM into four activities:

1. Acquisition of items to form a collection

2. Organization of items

3. Maintenance of the collection (store, edit, delete, archive)

4. Retrieval of items for reuse

The first activity, acquisition, can be done manually or automatically. Manual acquisition includes items users accumulate with their own actions at the time of their appearance in a digital environment (downloaded files from the Internet, personally created documents, etc.). Automatically gathered items enter the digital environment without user action or as a conse-

\author{
David A. Carr \\ Dept. of Computer Science and Electrical Eng. \\ Luleå University of Technology, \\ SE-971 87 Luleå, Sweden \\ david@sm.luth.se
}

quence of the previous actions that started automatic retrieval (new e-mail, subscriptions to RSS feeds, cached files of previously visited web pages, etc.). Manual or automatic acquisition can result in items having different value to the user [5].

The next activity, organization, includes classifying, naming, grouping, and storing items in a digital location. Good organization helps users find items faster. Studies have shown that there are diverse groups of users who organize their collections of files, e-mail, or bookmarks differently. The most common groups are: total filers (also called frequent or creation-time filers), occasional filers (spring or partial filers), and non-filers (also pilers) [5]. Each user can be in a different group for each collection and can switch groups over time.

Maintenance includes the usual activities: backing up the information space, updating out-of-date items, and archiving items that users feel they do not immediately need but might want to look at or use in the future.

The last and most critical activity, retrieval for reuse, depends heavily on actions and routines taken during the previous activities. Retrieval also depends on the situation and available information when it is initiated. There are different strategies and tactics for searching. Barreau [3] found that people most frequently use a browsing strategy that is initiated with a probe. This strategy allows users to get an overview of the collection while browsing and more importantly, provides clues to age, context, format, topic, and other dimensions of items encountered. Later studies confirm this behavior. Users navigated to items taking small steps using contextual knowledge as a guide rather than using a keyword search. This often occurred even when they knew the exact search target $[4,10]$. The authors call this technique "orienteering".

The rest of this paper continues with an indepth discussion of the problems users encounter when managing their personal information. 
We then propose a solution that should alleviate some of these problems.

\section{PIM Problems and Related Work}

PIM activities require great organizational skill and a great deal of time. Users usually do not want to spend this time and effort, especially on collections seen as unimportant or where they do not have a strong feeling of ownership. To cope with this, users develop their own methods for handling each type of digital information and each activity. We will take a closer look at each of them, describe problems that arose, strategies used, and research prototypes, where they are available.

\subsection{Acquisition to Form a Collection}

Three main collections are: the user's home directory, e-mail account(s), and web-browser bookmarks. Items are acquired automatically and manually in each collection. E-mail messages, for example, enter the user's inbox without user action. But, the sent-mail folder fills up as a result of user actions. Items enter the home directory and bookmarks primarily as a result of user actions. We believe that how an item is acquired affects its value.

There seem to be two main variables affecting the importance of items in a collection, their source and their time of acquisition. The more effort that the user spends on an item and the newer it is, the more important it is. The user feels a stronger sense of ownership.

This means files that users have created are generally more important than files they download from the Internet. The importance of items can change significantly during short time periods. With other items entering a user's digital environment, even the most important items can temporarily lose their importance. An email can be very important at the moment it appears in the inbox because it might contain instructions to handle an urgent matter. It can temporarily become more important than all other self-authored documents. But, it can lose that importance quickly after the instructions are completed.

A scale of relative importance is shown in Figure 1. Self-authored items have the highest value. Items with additional added value are items that were not originally the user's, but they acquired additional value due to the user spending time locating and/or editing them. At

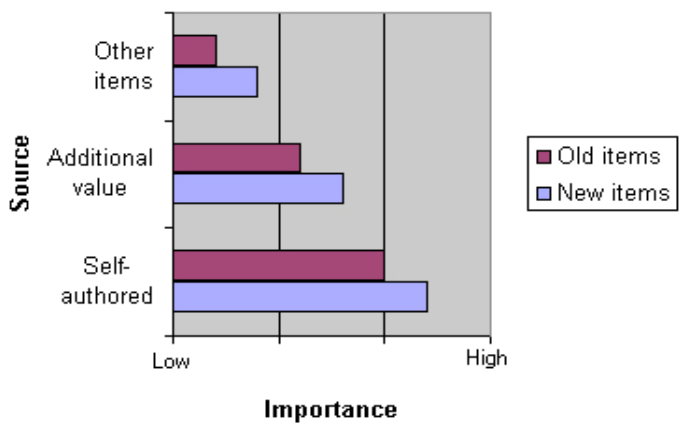

Figure 1: Relative item importance

the low end of the scale are items that enter a digital environment automatically where users do not spend any time creating, editing, or finding them.

An alternate classification [2] of acquired items is,

- ephemeral (short-lived information such as e-mail, calendar notes, to-do notes, etc.),

- working (frequently used and relevant to the user's work), and

- archived (long-lived information indirectly relevant to user's work).

In the long run, working and archival information have the highest importance to users since it contains self-authored items and items with added value.

\subsection{Organization of Items}

The primary organizational scheme provided by digital environments is a hierarchy. Current tools for file systems, e-mail, and bookmarks all utilize it. It is a fundamental method for formally dealing with complexity. However, there is no reason to believe it is always appropriate for digital documents.

Organization of documents in a hierarchy is a difficult task for most users [11, 14]. Classifying, naming, and labeling documents demands a huge cognitive effort. Self-created documents are generally filed hierarchically in the home directory. But, downloaded documents and e-mail (ephemeral information), are often left in the inbox or placed in some temporary place such as the desktop or a temporary folder. Users tend to leave them there for later classification or as a reminder.

When surveyed about PIM-tools problems users reported that [14],

- the separation of files, e-mail, and bookmarks is inconvenient since together they form a complete digital information space; 
- there is no support for short-term notes or remarks, and available solutions are not practical or useful; and

- there is no link between various resources.

Users usually group items by thematic proximity and document type. In file systems users create folders for three to seven items on the same subject. While on the desktop, they organize document icons and folders in rectangles, circles, and lines as an aid to speed-up search. Thematically similar items reside in all three collections. One would think that many folders will overlap, but Boardman and Sasse [5] found that most folders in different hierarchies do not overlap, and that users may have different organizational needs for each collection. Automatic replication of folders in all collections might also lead to confusion and cause users to attempt to retrieve an item from the wrong collection (especially with several email accounts). Boardman and Sasse also reported that users tend to spend more time organizing files and do it more extensively than for e-mail and bookmarks. They suggest that,

- users spend more time organizing files because they feel stronger ownership;

- users think that file re-use is more likely than the re-use of e-mail or bookmarks;

- files and bookmarks are created incrementally and are easier to organize than e-mail; and

- personal factors (e.g., tidiness), might affect organizational habits.

User problems with hierarchies have led researchers to experiment with alternatives. Ravasio, et. al. [14] find hierarchies a rigid and unnatural storage data structure for digital information. Other problems seen in PIM tools include [6]:

- Files can only be at one place at a time.

- File-organizational structure cannot easily change over time.

- Dynamically organized collections (according to projects, time, content, etc.) are not possible.

- Automation in organization is lacking.

In order to solve these problems, researchers have developed methods that are extensions of present tools. However, others have completely abandoned the desktop metaphor and hierarchies to bring new approaches to PIM.

Interaction in "Presto" [6] is based on document attributes. Numerous attributes hold meaningful document information. By forming queries on these attributes, users create dynamic document sets. To get the most from Presto, users must add correct attributes to every item. This requires the same or even larger organizational skills than for hierarchal structures. A poorly formed system would produce useless sets. However, the concept of dynamic collections and different document attributes for different users could improve usage and increase PIM flexibility

"Lifestreams" [8] is another non-hierarchal prototype that presents documents in chronological order. The system stores all created, modified, and received documents on a timetable. In addition, items can be manually placed in the future as a reminder. "MyLifeBits" [9] also uses time as the main organizational aid. It supports four different views: A timeline view where documents about an event are displayed at the time the event occurred. A clustered-time view groups together documents by similar time. A detailed view displays a list of resources, and a thumbnail view that displays thumbnails in a grid. Multiple views give users different perspectives on their collections.

The "Umea" [10] system tries to place itself above present PIM tools. It allows users to create new projects, and all activities (creating documents, reading emails, visiting web pages) are stored under the selected project. Hence, all links to items related to one project are stored in one location.

Another automated system for document classification was presented with the "electronic file cabinet" [12]. It can fill in documents automatically, remember, organize, update, and help users find the desired documents in the real world. Each scanned and OCR-ed document is compared with the rest of the database to find its multiple filing locations.

\subsection{Maintenance}

Maintenance covers all changes in the digital information space: changing the structure of hierarchies, renaming items and folders, moving them, deleting unused and unnecessary items, making backups, etc.

In early studies of PIM behavior, users did not create archives [2] because there was not enough storage space. This is no longer a concern, and users see archiving as important since they put a lot of effort into creating and labeling storage structures [14]. Maintenance focus is primarily on the file system, but users also 
maintain e-mail and bookmark hierarchies. In Ravasio, et. al. [14], the age of archived files ranges from one-half to eight years, and users regularly returned to their archives. They found that users stored files on the desktop in addition to their main hierarchy. Eventually, limited desktop space led to a shortage if users continuously stored new items on it. Over time users separated items into three categories:

1. Important items that remained in place

2. Valuable things that moved to the archives

3. Unimportant items that were deleted.

Items are similarly managed in the inbox.

There does not seem to be good support for archiving in any PIM tool. Some file-system browsers can delete old and unused items, but they can do a lot of damage if users are not alert. Some e-mail clients store old, sent items by month or delete them after a certain age. Otherwise, maintenance is left to the user.

\subsection{Retrieval of Items for Reuse}

Users can search two spaces for information, their personal computer (including files and e-mail on a local server), and the Web. While users are familiar with their own information space, they are unfamiliar with the vast number of online documents. Therefore, they use different primary search strategies, orienteering (sorting and browsing), for their personal information space and teleporting (search engines), for the Web [4]. Even though search engines are effective, they are not preferred in the personal information space. Search engines require as much cognitive and mechanical effort as orienteering, but orienteering is preferred because it helps users to "... brush-up their knowledge of the general organization of their information space while searching [14]." Search is primarily used as a tactic if orienteering fails.

Visually searching items on the desktop is easy since its surface provides an overview of items stored on it, and there are no problems finding them. Unfortunately, there is not enough space to store a large number of items.

The inbox is the temporary storage space for e-mail. It can contain hundreds of items, and users primarily use sorting to search it. Email programs provide sorting by subject, sender, date, importance, attachments, etc. These attributes are actually closer to the way people think about their documents [14].
While orienteering, users employ all of the contextual knowledge that they have about the target item [4, 11]. Contextual knowledge helps them position the item in the information space and dig it out afterwards.

Problems users have when retrieving information in their personal environment are:

- Technical document metadata such as filename and path do not help in searching.

- Users understand files characteristics differently than the system does.

- All search tools require users to remember the documents by their names and types, which is different from the user's contextual understanding of the documents.

- The position of the document in the hierarchy is drawn from the context in which the document was created, used, and filed. Present tools cannot provide this context.

- Current search tools are not easy to operate. They require some experience to manage and use them in a way that achieves reliable results. They lack the ability to search by content, independent of file format.

As a response to all these well-known problems Dumais et. al. [7] created a tool called "Stuff I've Seen" or "SIS". They designed a tool that provides unified access to items (email, web pages, documents, etc.), that the user has already seen. While searching, rich contextual information is given. This is based on the premise that users primarily retrieve items that they have already seen, and that rich contextual information will simplify searching for them.

The web is a large information space changing all the time, and users tend to teleport to items more often than they do in their personal information space. In addition web search tools are more sophisticated than those for e-mail and files. But even on the web, users try to orienteer whenever possible. Users looking for a university phone number would go to the university's web page and find it there rather than use a search engine. As with e-mail and files, users teleport when they cannot find any contextual link to the information or when orienteering is not successful.

\section{Suggestions for Improvement}

Each of the above systems solves some of the problems encountered in the four PIM activities, but none of them is a complete solution. A complete solution must, 
- support management of multiple hierarchies and building relationship maps among items,

- allow limited duplication of items in the personal information space,

- support extra attributes for items,

- provide automatic organization support in order to help users build relationship maps,

- simplify search tools, and

- provide contextual information to support orienteering.

We propose a PIM system that supports the natural system that many people use for organization of paper documents - piles of documents [13]. Document piles that we compose in real life contain documents from various sources. We might collect photos, newspaper articles, our own notes, and other documents on a particular subject. What if we provide a similar but even more powerful possibility in the digital environment? What users actually collect in their information space is the content of their documents - not icons, filenames, and paths. In our system, the organizing feature will be piles of thumbnails (Figure 2) rather than windows and icons. The aim of scattered thumbnails is showing content while managing information.

Items would be organized by just draggingand-dropping them onto a pile. Users might drag in an entire document, selected text from a web site, a photo, an audio file, an e-mail message, an extract from a conversation whatever they think is relevant to that pile. They would see all this information in thumbnails regardless of source, size, and format. Users who want a more orderly structure would be supported by allowing replacement of piles by labeled folders.

In addition to thumbnails, piles could have a

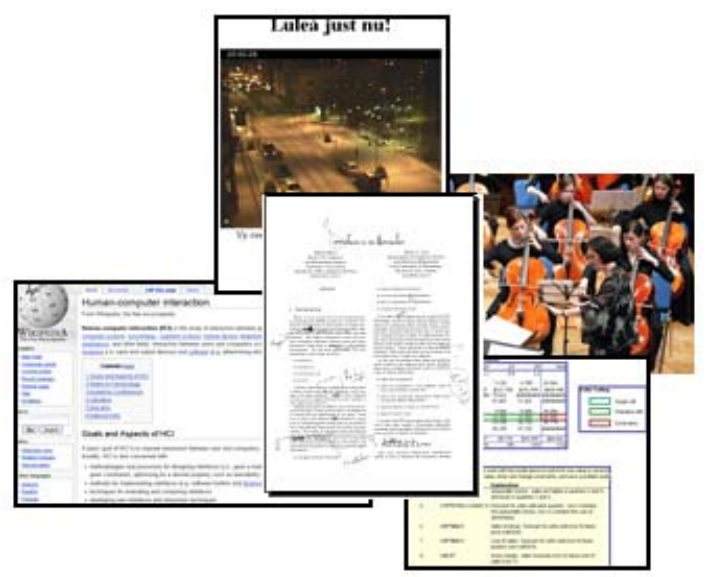

Figure 2: Pile of thumbnails name and a detailed description. These extra attributes might be generated automatically based on the pile's content. The system could extract names, titles, and similar information to provide even richer clues about a pile and its contents. Users too, might add attributes. But if attributes are gathered automatically, it is easier for the user to just change them. This extra information, together with traditional attributes, such as filenames, formats, and modification dates, could be shown in a popup label (Figure 3).

If desired, the system could help users create piles based on collected attributes. While creating a new pile, users might ask the system if there are other items in their information space that could be relevant to new pile. But, piles would always be manually created. Automatically created piles would not provide contextual clues and would not mean much to users.

Items on the desktop are easiest to find. Scanning a desktop with an average number of piles and thumbnails on it can provide quick results. With a larger number of piles, an infinite desktop such as those provided by zooming interfaces [1] would support orienteering. In addition, an automatic search tool that provides fast visual results on the desktop would be used. Changing the criteria in the search tool would change the border color of thumbnails matching those criteria dynamically and quickly get user's attention. Matching thumbnails might even be moved to the front of the document pile and enlarged. Sliders and other direct manipulation widgets would be used to provide a dynamic-query interface to the documents [15].

Users might combine document piles, or they could copy items from a pile and place them in another. Most recently used items would be on top.

This proposal supports the requirements for PIM solutions in the following ways:

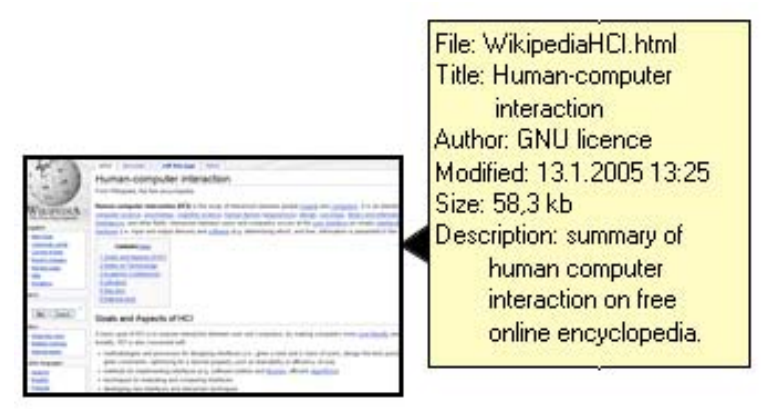

Figure 3: Thumbnail label 
- The piles allow users to organize documents, and relationships among piles can be expressed by placing them near each other.

- Items can be duplicated by placing copies in different piles.

- Both manually and automatically generated attributes can be associated with document piles.

- The system assists in building new document piles by locating items with similar attributes.

- Visual search and automatic search with real-time visual feedback simplify item location.

- Items are always displayed in the context provided by the pile metaphor.

\section{Conclusion}

This paper presents a survey of personal information management research. From this survey we see that users still have problems. A fundamental problem seems to be that PIM systems are still organized to simplify implementation and not to support the user's way of working. We propose to correct this by supporting a pile-of-documents metaphor, which emulates a common method for handling physical documents.

Understanding all the problems of present tools is just the beginning of a long journey toward improved PIM tools. Our immediate goal is to implement our ideas in order to test them and to refine our design.

\section{Acknowledgements}

We would like to thank Carl Rollo for proofreading drafts of this paper. He contributed greatly to the paper's readability.

\section{References}

[1] Bederson, B.B., Meyer, J., Good, L., Jazz: an extensible zoomable user interface graphics toolkit in Java, Proceedings of UIST 2000, CHI Letters, 2(2), 6-8 Nov 2000, San Diego, CA, 171-180.

[2] Barreau, D. K., Nardi. B. A., Finding and reminding: file organization from the desktop. SIGCHI Bulletin, 27(3), July 1995, 39-43.

[3] Barreau, D. K., Context as a factor in personal information management systems. J. Am Soc. Inf. Sci., 46(5), 1995, 327-339.
[4] Bergman, O., Boardman, R., Gwizdka, J., Jones, W., Personal information management. SIGCHI 2004 Extended Abstracts, 24-29 April 2004, Vienna, 1598-1599.

[5] Boardman, R., Sasse, M. A., Stuff goes into the computer and doesn't come out: a cross-tool study of personal information management. Proceedings of SIGCHI 2004, 24-29 April 2004, Vienna, Austria, 583-590.

[6] Dourish, P., Edwards, W. K., LaMarca, A., Salisbury, M., Presto: an experimental architecture for fluid interactive document spaces. ACM Trans. on Computer-Human Interactions, 6(2), March 1999, 133-161.

[7] Dumais, S., Cutrell, E., Cadiz, J. J., Jancke, G., Sarin, R., Robbins, D. C., Stuff I've seen: a system for personal information retrieval and re-use. Proceedings of ACM SIGIR 2003, 28 July - 1 Aug 2003, Toronto, Canada 72-79.

[8] Fertig, S., Freeman, E., Gelernter, D., Finding and reminding reconsidered. SIGCHI Bulletin, 28(1), Jan 1996, 66-69.

[9] Gemmell, J., Bell, G., Lueder, R., Drucker, D., Wong, C., Mylifebits: fulfilling the MEMEX vision, Proceedings of MULTIMEDIA'02, 1-6 Dec 2002, Juanles-Pins, France, 235-238.

[10] Kaptelinin V., Umea: translating interaction histories into project contexts. Proceedings of CHI '03, 5-10 April 2003, Ft. Lauderdale, FL, 353-360.

[11] Lansdale, M., The psychology of personal information management, Applied Ergonomics, 19(1), 55-66, 1988.

[12] Lawrie, D., Rus, D., A self-organized file cabinet. Proceedings of the Eighth International Conference on Information and Knowledge Management, 2-6 Nov 1999, Kansas City, MO, 499-506.

[13] Malone, T. W., How do people organize their desks?: implications for the design of office information systems. ACM Trans. on Information Systems, 1(1), Jan 1983, 99-112.

[14] Ravasio, P., Guttormsen-Schär, S., Krueger, H., In pursuit of desktop evolution: User problems and practices with modern desktop systems. ACM Trans. Computer-Human Interaction, 11(2), June 2005, 156-180.

[15] Shneiderman, B., Dynamic queries for visual information seeking, IEEE Software, 11(6), Nov/Dec 1994, 70-77. 